\title{
Sources of auditory masking in infants: Distraction effects
}

\author{
LYNNE A. WERNER and JLL Y. BARGONES \\ University of Washington, Seattle, Washington
}

\begin{abstract}
Previous work has demonstrated that infants' thresholds for a pure tone are elevated by a masker more than would be predicted from their critical bandwidths. The present studies explored the nature of this additional masking. In Experiment 1, detection thresholds of 6-month-old infants and of adults for a $1-\mathrm{kHz}$ tone were estimated under three conditions: in quiet, in the presence of a 4- to $10-\mathrm{kHz}$ bandpass noise at $40 \mathrm{~dB}$ SPL, and in the presence of the same noise at $50 \mathrm{~dB}$ SPL. The noise was gated on at the beginning of each trial. Adult thresholds were the same in all three conditions, indicating that little or no sensory masking took place in the presence of the noise. Infant thresholds were about $10 \mathrm{~dB}$ higher in the presence of the noise. We term this effect distraction masking. In Experiment 2, the effect of gating the noise on at trial onset was examined. Thresholds for the same tone were estimated in quiet and in the presence of the bandpass noise at $40 \mathrm{~dB}$ SPL, but the noise was presented continuously during the session. Under these conditions, distraction masking was still observed for infants. These findings suggest that a masker can have nonsensory effects on infants' performance in a psychoacoustic task.
\end{abstract}

Masking has proved to be a powerful psychophysical paradigm for investigating the basic properties of sensory systems, and in recent years several investigations into the development of auditory masking have been published. Masked thresholds are often reported to be elevated in infants and children relative to adults (e.g., Allen, Wightman, Kistler, \& Dolan, 1989; Nozza \& Wilson, 1984; Schneider, Trehub, Morrongiello, \& Thorpe, 1989), but this is not always the case, at least in older children (Veloso, Hall, \& Grose, 1990). Even when the masked threshold is elevated, the amount of masking (i.e., the difference between masked and unmasked thresholds) is not always greater in younger subjects (Nozza \& Wilson, 1984; Schneider et al., 1989).

Olsho (1985), however, reported a case of tone-on-tone masking in 6-month-old infants where the amount of masking exhibited by the infants was about $14 \mathrm{~dB}$ greater than that exhibited by adults under the same condition. The purpose of the Olsho study was to estimate psychophysical tuning-curve widths of infants and adults. The level of a .5-, 1-, 2-, or 4-kHz tone, the probe, was set at $25 \mathrm{~dB}$ sensation level (SL). Thresholds for the probe in the presence of a second masking tone were obtained for three different masker frequencies to measure tuning-

This research was supported by NIH Grant DC00396 to L. A. Wemer (formerly L. W. Olsho). Preliminary results of the studies were reported at the Midwinter Research Meetings of the Association for Research in Otolaryngology in 1989 and 1990 . We thank Pat Feeney, Cam Marean, Lisa Rickard, and Heather Taylor for assistance in data collection. John Palmer and Cam Marean also made helpful comments on the manuscript. Requests for reprints should be addressed to Lynne Werner, Child Development and Mental Retardation Center, WJ-10, Box 47, University of Washington, Seattle, WA 98195. curve width, or $Q_{10}$. Masker level was manipulated to define the threshold. At every masker frequency and every probe frequency, the infants exhibited masking at a masker level ranging from 11 to $16 \mathrm{~dB}$ lower than the level at which the adults exhibited masking. On the average, then, infants showed $14 \mathrm{~dB}$ more masking than did the adults. We refer to this additional masking shown by infants, relative to adults, as excess masking.

Under traditional interpretation, the masked threshold is a measure of the frequency selectivity of the auditory system (Fletcher, 1940). Patterson, Nimmo-Smith, Weber, and Milroy (1982), however, demonstrated that two factors influence the masked threshold: the selectivity and the processing efficiency of the system. Efficiency is defined as the signal-to-noise ratio at the output of the auditory filter required to achieve a given detection performance. A 3-dB difference between two listeners in masked threshold, they argued, could result from either of these factors: One listener could have an auditory filter twice as wide as the other, or that listener could be less efficient than the other. In this example, the less efficient listener would require a $3-\mathrm{dB}$ higher signal-to-noise ratio to detect the signal.

One explanation for excess masking, then, is that the infants have wider auditory filters than adults do. This wide-filter hypothesis fails as a general explanation for excess masking on two grounds. First, in some conditions (e.g., a pure-tone masker $50 \mathrm{~Hz}$ above the pure-tone probe, as in Olsho, 1985), excess masking is observed when filter width could not be the limiting factor in probe detection, because both masker and probe fall entirely within the same filter. Second, the major finding of OIsho was that infants and adults did not differ in psychophysical tuning-curve width. Schneider, Morrongiello, 
and Trehub (1990) have subsequently shown that 6-montholds' critical bandwidths do not differ substantially from those of adults.

The other explanation for excess masking in infants is processing inefficiency or a higher criterion signal-tonoise ratio within the filter for detection of the signal. In the limit, efficiency is determined by the precision of intensity coding in the auditory system. Certain aspects of neural intensity coding, including dynamic range and maximum discharge rate, are known to mature in the period following onset of cochlear function in mammals (reviewed by Sanes \& Rubel, 1988). There are also a few studies in which poorer intensity discrimination has been reported among infants and children (Jensen, Neff, \& Callaghan, 1989; Sinnott \& Aslin, 1985), and Schneider et al. (1989) have argued that changes in intensity coding could account for age-related changes in masked thresholds in early development. Although this hypothesis remains tenable, it has not been tested. Furthermore, masking differences persist in humans at ages far older than one would predict from the physiological data on intensity coding obtained from other mammals.

Factors other than intensity coding are known to affect listener criterion (see, e.g., Green \& Swets, 1966), but the role of such factors in the development of psychophysical performance has not been studied. Attention is one factor commonly cited as a potential contributor to the infant's poor performance in some psychoacoustic tasks. Although attention is only vaguely defined in most cases, there is a specific way in which attention could contribute to excess masking. In order to detect a probe in the presence of a masker, a listener not only must be able to physically isolate the relevant spectral information, but also must selectively process that information. Under conditions of uncertainty, even adults may have difficulty directing attention to the appropriate cue (e.g., Neff \& Green, 1987; Watson, Kelly, \& Wroton, 1976). One explanation for excess masking in infants, then, is that even though infants' auditory systems provide them with precise spectral information, they are less able than adults to selectively attend to the information representing the signal or probe.

In the present study, we tested the hypothesis that excess masking in infants can result from immature attention. Thresholds for a pure tone at $1 \mathrm{kHz}$ were measured in quiet and in the presence of another sound that was not expected to produce any peripheral interference with the tone. We termed this other sound a distraction masker because we predicted that it would compete with the tone for attention, even though it would be processed by different peripheral channels. The distraction masker was a bandpass noise, with frequency cutoffs of 4 and $10 \mathrm{kHz}$. This sound would not be expected to produce masking of the $1-\mathrm{kHz}$ tone for two reasons: First, higher frequency sounds tend to be ineffective maskers of lower frequency sounds (see, e.g., Wegel \& Lane, 1924). Second, the auditory filter around the $1-\mathrm{kHz}$ tone would have to be ex- tremely wide to pass any energy in regions $3 \mathrm{kHz}$ or more away from it. Patterson et al. (1982), for example, report equivalent rectangular bandwidths around $1 \mathrm{kHz}$ to be $130-200 \mathrm{~Hz}$ in adults, and, as described above, the existing data for 6-month-old infants suggests that they do not differ from adults in auditory filter width.

\section{EXPERIMENT 1}

\section{Method}

Subjects. A total of 466 -month-olds (average age 185 days, range 166-195 days) were tested. Thirteen infants were excluded because they failed tympanometric screening on the test day. The final sample of infants included 9 who completed testing in at least two conditions. Four of the infants failed to reach training criteria on repeated visits; the other 20 did not provide complete data sets because of insufficient number of trials or variable responses in at least one condition. Eleven 18- to 28-year-old actults also participated; 1 failed the tympanometric screen. The final sample of adults included 6 subjects who completed testing in all conditions. Two of the adults were excluded because of experimenter errors, 1 had unacceptably variable reversals, and 1 drove the stimulus level into the noise floor. All subjects were healthy on the test date, with no family history of congenital hearing loss, no personal history of hearing dysfunction, no more than two prior episodes of ear infection, and no ear infection within 3 weeks prior to testing.

Stimuli. A 1-kHz pure tone, $500 \mathrm{msec}$ in duration with $16-\mathrm{msec}$ rise/fall time, was the signal. On each presentation, this stimulus was repeated 8 times with $500 \mathrm{msec}$ between repetitions. The distraction masker was a bandpass noise, filtered at $90 \mathrm{~dB} /$ octave with cutoffs at 4 and $10 \mathrm{kHz}$. The masker was $8 \mathrm{sec}$ in duration, with $16-\mathrm{msec}$ rise/fall, and was gated on with the first repetition of the signal on signal trials in masked conditions. Stimuli were presented via Etymotic ER-1 insert earphones in trimmed foam ear tips. Testing was conducted in a double-walled sound-attenuating booth.

Threshold for detecting the $1-\mathbf{k H z}$ signal was measured for each subject in three conditions: in quiet, with the distraction masker at an overall level of $40 \mathrm{~dB}$ SPL, and with the distraction masker at an overall level of $50 \mathrm{~dB}$ SPL. In the quiet condition, the signal was presented on signal trials, and no stimulus was presented on no-signal trials. In the two masker conditions, both the signal and the distraction masker were presented on signal trials, and only the masker was presented on no-signal trials. The order of conditions was counterbalanced across subjects.

Procedure. Infant responses to the signal were measured with the observer-based psychoacoustic procedure (Olsho, Koch, Halpin, \& Carter, 1987), which has been described in detail previously (Olsho, Koch, \& Halpin, 1987; Olsho, Koch, et al., 1987; Spetner $\&$ Olsho, 1990). The basic logic behind the procedure is that if an observer who is blind to trial type can distinguish signal from nosignal trials solely on the basis of the infant's behavior, then the infant must be able to hear the signal. The infant's responses to signals are reinforced by the activation of a mechanical toy bear. The infant is held by a parent during the session. An assistant in the test booth keeps the infant facing toward a video camera and observation window by manipulating simple quiet toys. Neither of these adults can hear the sounds presented to the infant: the parent listens to music over circumaural headphones, and the assistant monitors activity in the control room over headphones to determine when trials are in progress and to communicate with the observer.

A session in the present experiment had three phases. In the first phase, the probability of a signal trial was 0.75 , and the mechanical toy reinforcer was activated following a signal trial-whether or not the observer responded correctly. Signal level was $75 \mathrm{~dB}$ 
SPL. This phase continued until the observer responded correctly on four of five consecutive trials and had at least one correct rejection. In the next phase, the probability of a signal trial was 0.5 , and the reinforcer was activated only when the observer responded correctly on a signal trial. Signal level was $75 \mathrm{~dB}$ SPL. This phase continued until the observer responded correctly on four of five consecutive signal trials and four of five consecutive no-signal trials. In the third phase, threshold was estimated adaptively. The probability of a signal trial was 0.5 , and the reinforcer continued to be activated only when the observer scored a hit. The level of the signal on the first trial was $70 \mathrm{~dB}$ SPL. Step size began at $10 \mathrm{~dB}$ and varied following PEST rules (Taylor \& Creelman, 1967). If the observer was correct (hit or correct rejection) on two consecutive trials, the level of the signal was reduced by one step. If the observer was incorrect on one trial, the level of the signal was increased by one step. Testing continued until at least eight reversals had occurred. Threshold was taken as the average of the last six reversals. The standard deviation of the reversals of an acceptable threshold was less than $5 \mathrm{~dB}$. Examples of trial-by-trial protocols for an infant in two conditions are given in Figure 1.

\section{Quiet Condition}
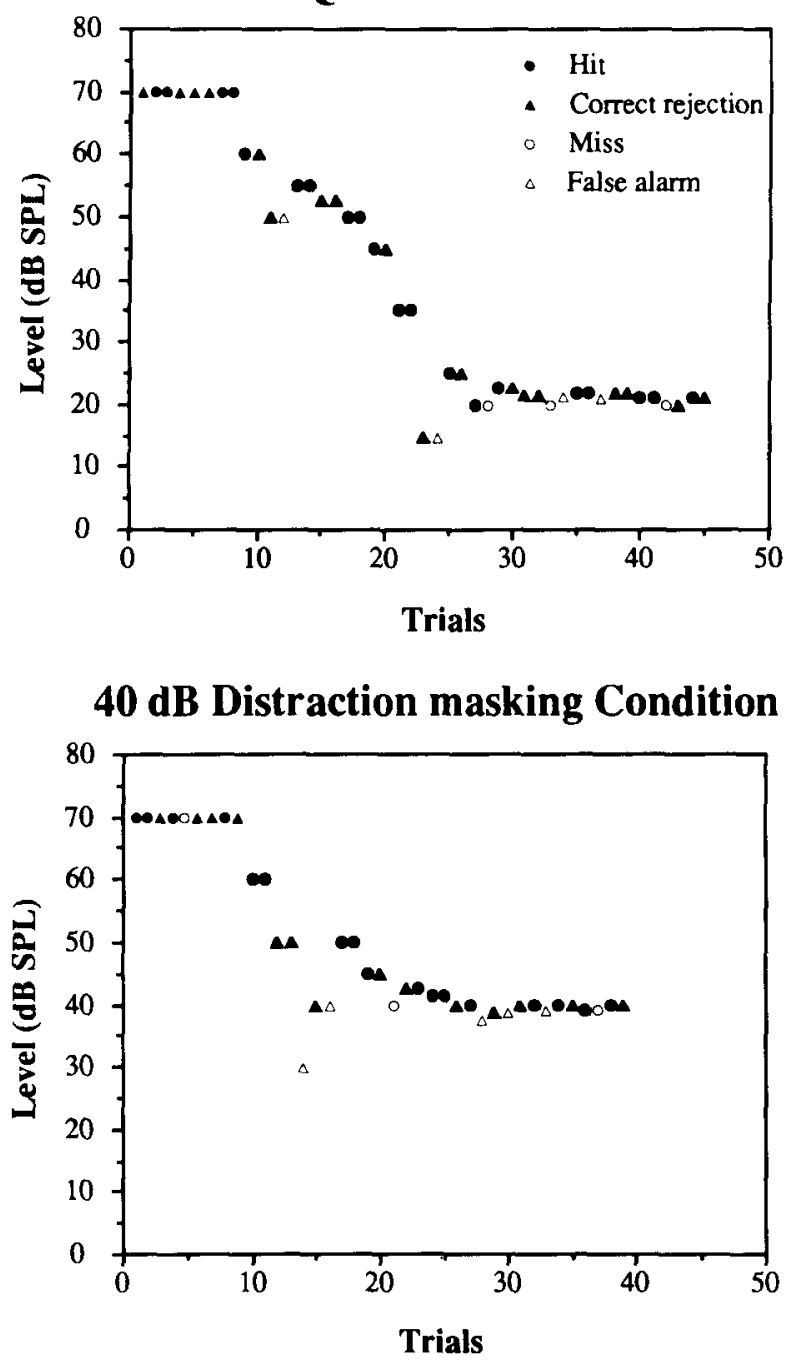

Figure 1. Examples of trial-by-trial protocols obtained from a 6 month-old in the quiet and in 40-dB distraction-masking conditions.

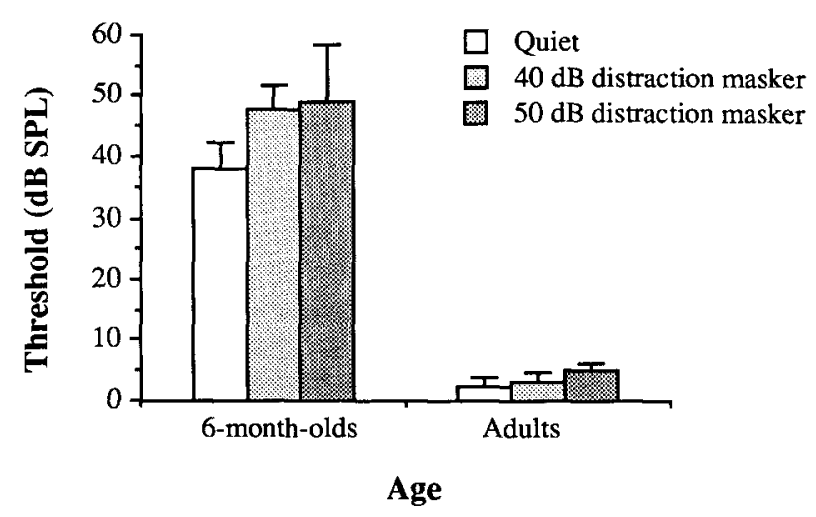

Figure 2. Average thresholds for 6-month-olds and adults in three conditions in Experiment 1. Error bars represent $\pm 1 S E M$.

The test method for adults was as similar as possible to the test method for infants. They listened alone in the test booth and raised their hands whenever they "heard the sound that makes the bear come on." All other aspects of the psychophysical procedure were identical.

\section{Results}

Average thresholds. Only 4 of the 26 infants who produced at least one threshold produced thresholds in all three conditions. In fact, it was inordinately difficult to obtain infant thresholds in the 50-dB distraction-masker condition. The mean thresholds of the adults and the infants who provided thresholds in quiet and in at least the 40-dB distraction-masker condition are shown in Figure $2 .^{1}$ Adult threshold increased only slightly-less than $2 \mathrm{~dB}-$ in the presence of either masker. Infant threshold, on the other hand, was about the same in the 40- and 50- $\mathrm{dB}$ distraction-masker conditions, but about $10 \mathrm{~dB}$ higher than it was in quiet.

Because the variance was so much higher in infant thresholds than in adult thresholds, infant and adult data were analyzed separately. A one-way repeated measures analysis of variance of the adult thresholds in three conditions showed no significant effect of condition $[F(2,9)$ $=1.43, p=.3$ ]. A one-tailed paired samples $t$ test between infant threshold in quiet and infant threshold in the 40- $\mathrm{dB}$ distraction-masker condition, however, was significant $[t(8)=-1.93, p<.05]$. Thus, for infants, the presence of a second sound-even one that would not be expected to produce peripheral interference-still had the effect of increasing the detection threshold, as would be predicted by an attentional hypothesis about the effects of maskers on infants' thresholds.

\section{EXPERIMENT 2}

As noted in the introduction, not all studies of masking in infants have resulted in excess masking effects (Nozza \& Wilson, 1984; Schneider et al., 1989). One difference between those studies and both Olsho (1985) and Experiment 1 is that the studies that yielded excess masking involved maskers that were gated on at the beginning of each trial, whereas the studies that did not yield the ef- 
fect involved maskers that were on continuously throughout the session. One might argue that when the masker is on continuously, the infant "learns" to ignore the masker and thus does not show distraction effects. In order to address that issue, we repeated Experiment 1 using a continuous masker.

\section{Method}

Subjects. A total of 1306 -month-olds (average age $=187$ days, range $=181-197$ days) participated. Twenty-nine infants failed the tympanometric screen. The final sample consisted of 20 infants who provided a complete data set; of the remaining infants, 12 never met training criteria, 66 provided insufficient or variable data, and 3 were excluded because of experimenter error. Thirteen 20 - to 30-year-olds also participated. All adults passed tympanometry, but two data sets were excluded because of experimenter error, leaving 11 adults in the final sample. All subjects met the criteria for inclusion described for Experiment 1.

Stimuli. The signal was the same 1-kHz tone as in Experiment 1. The masker was the same bandpass noise. The masker was turned on at the beginning of the session, and it remained on throughout.

Each subject was tested in quiet and with the distraction masker at an overall level of $\mathbf{4 0} \mathrm{dB}$ SPL. Signal and no-signal trials in quiet were defined as in Experiment 1. Signal trials in the distractionmasker condition consisted of eight repetitions of the tone; no-signal trials were 8-sec periods when no signal was added to the noise. The order of conditions was counterbalanced across subjects.

Procedure. The same test method, the observer-based psychoacoustic procedure, was used. Two changes were made in the procedure. In the second training phase of the experiment, four different randomly ordered signal levels were used, ranging from $20 \mathrm{~dB}$ below the starting level of the adaptive run to the starting level of the adaptive run. We had found in other experiments that variable training levels were helpful in training 6-month-olds (e.g., Werner \& Marean, in press). In addition, the starting level of the adaptive run was reduced on the basis of the thresholds obtained in Experiment 1 . To make sure that the thresholds in quiet were not overestimated because the starting level was too far above the threshold, for one group of infants the starting level was $50 \mathrm{~dB}$ SPL. Another group of infants was tested with a starting level of $65 \mathrm{~dB}$ SPL in the quiet condition. The starting level for both groups in the distraction-masker condition was $65 \mathrm{~dB}$ SPL. Other aspects of the psychophysical procedure were the same as in Experiment 1.

\section{Results}

Mean threshold for the tone in quiet was compared for the two groups of infants tested at different starting levels. An independent groups $t$ test indicated that the difference between these means was not significant $[t(18)=-0.65$, $p=.52$ ]. For the remaining analyses, the data of both groups of infants was considered without regard to starting level in quiet.

The average thresholds in the quiet and distractionmasker conditions for infants and adults are shown in Figure 3. Threshold for the signal in the distractionmasker condition was about $5 \mathrm{~dB}$ higher for the infants, and about $2 \mathrm{~dB}$ lower for the adults, compared to the threshold in quiet. Because the infants' data were so much more variable than the adults', statistical analyses were conducted separately for the two groups. One-tailed paired samples $t$ tests showed that the infants' thresholds were significantly higher in the distraction-masker condition $[t(19)=-1.90, p=.04]$. The difference between the

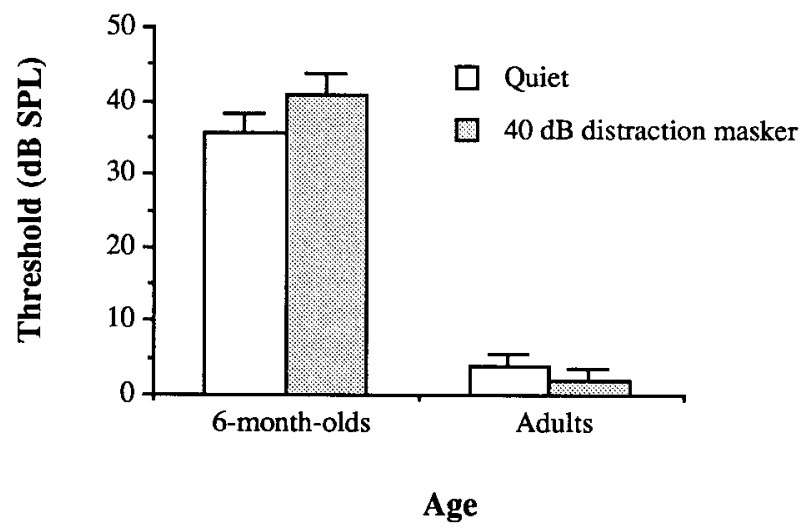

Figure 3. Average thresholds for 6-month-olds and adults in two conditions in Experiment 2. Error bars represent \pm 1 SEM.

thresholds in the two conditions for the adults was not significant. $^{2}$

The average amount of distraction masking found for the infants when the masker was continuous was about $5 \mathrm{~dB}$; in the case of the gated masker in Experiment 1, it was about $10 \mathrm{~dB}$. To test whether this difference was significant, we compared the infants who heard a gated masker at $40 \mathrm{~dB}$ SPL (Experiment 1) to those who heard a continuous masker at the same level (Experiment 2), using analysis of variance with repeated measures on the quiet versus distraction-masker factor and gated versus continuous as a between-subjects factor. Although the effect of having a distraction masker present was highly significant $[F(1,27)=7.96, p=.009]$, neither the main effect of gating the masker nor the interaction between gating and distraction masking was significant $[F(1,27)$ $=1.31, p=.26$, and $F(1,27)=0.70, p=.41$, respectively]. Thus, there is no statistical evidence that the amount of distraction masking observed with a continuous distraction masker is less than the amount observed with a gated distraction masker.

Signal detection analysis of infant performance near threshold. In a one-interval psychophysical paradigm like that used here, thresholds could be affected by both sensitivity and response bias. Thus, the effect of the distraction masker could be either to make the infant less sensitive to the signal or to change the infant's response criterion. In fact, it would not be hard to imagine that the infant might respond at a high rate on both signal and nosignal trials in the distraction-masker conditions.

To get a general idea of whether or not response bias varied with masking condition, we performed a receiveroperating characteristic (ROC) analysis of infant data. To ensure that the infant/observer team was operating in a fairly stable manner, we examined only trials from the adaptive phase of sessions and only trials that occurred after the staircase had converged on threshold. Only trials within $5 \mathrm{~dB}$ of the threshold were included. Hit and false alarm rates on these trials were calculated for each infant in each condition. The number of trials available for anal- 


\section{Gated distraction masker}

- Quiet

- $40 \mathrm{~dB}$ distraction masker

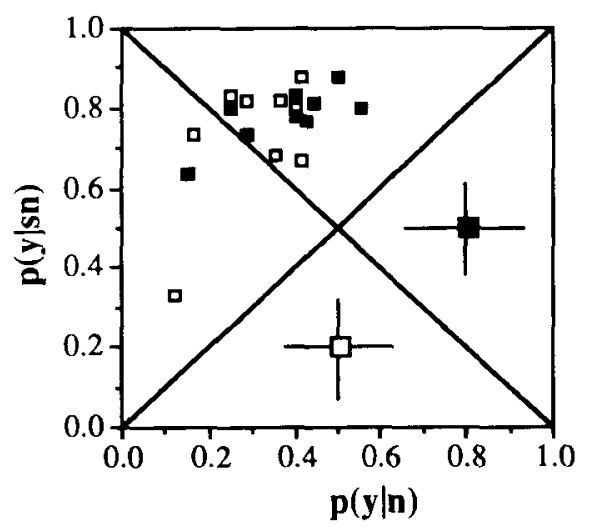

Continuous distraction masker

- Quiet

- $40 \mathrm{~dB}$ distraction masker

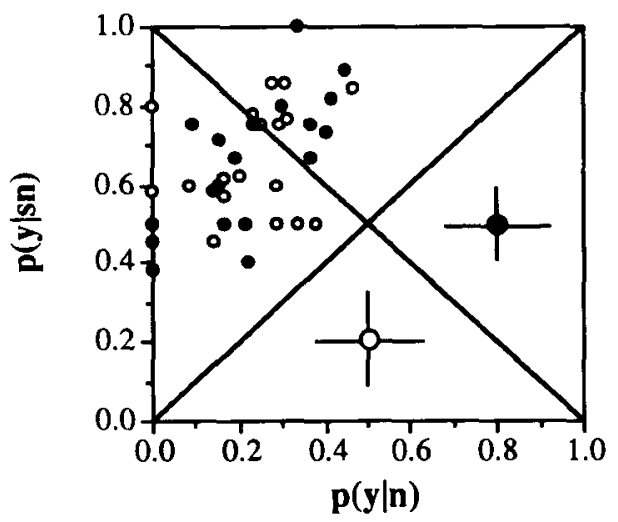

Figure 4. Receiver-operating characteristic plots of performance on trials near threshold for infants in two experiments. Each point represents the performance of an individual infant. $p(y \mid s n)$ is the probability of a "yes" response on a signal trial, or hit rate. $p(y \mid n)$ is the proportion of "yes" responses on no-dignal trinls, or false alarm rate. Crossed error bars in the lower part of each figure represent \pm 1 average binomial $S D$ in hit and false alarm rates in each condition.

ysis was typically about 25 . The hit and false alarm rates obtained are plotted in ROC space in Figure 4.

There is some variability in response bias, as indicated by the position of the plotted points along the dimension parallel to the positive diagonal. Keep in mind, though, that in order to account for the shift in threshold between the quiet and distraction-masker conditions, one would have to find a difference in criterion between the two conditions. In fact, there does not seem to be any difference between the quiet and distraction-masker conditions, either in the general position of the data points or in the vari- ability in position in either experiment ${ }^{3}$ It appears that observers tended to be generally lax in response criterion in the gated-masker condition and generally strict in response criterion in the continuous-masker condition. An explanation for this finding is not readily apparent. In any case, one would have to conclude from this analysis that the threshold elevation observed under conditions of distraction masking does not result from a shift in response criterion. Rather, it suggests that the attentional effect is to reduce sensitivity to the signal.

Other data bearing on the nature of distraction effects. Although ROC analysis suggests that the effect of the distraction masker was not simply to increase the infant's tendency to respond, there are other ways in which the additional sound may have affected the infant in a general way, without specifically affecting auditory selective attention. For example, thresholds in the distractionmasker conditions could be higher because the infant's level of motor activity was increased or because the noise was disturbing to the infant.

Although we do not have direct evidence bearing on infant activity level during the session, data do suggest that this type of general effect is not responsible for the threshold shift. For example, the trial-by-trial protocols (e.g., Figure 1) in quiet and distraction-masker conditions are both qualitatively and quantitatively similar. The average standard deviation of the reversal points used to estimate infant thresholds was the same in all conditions and in both experiments (Table 1). Furthermore, one might predict that if the effect of the masker was to make the infant "antsy," then the number of trials required to meet training criterion would be greater in the distractionmasker conditions. As can be seen in Table 1, there was a slight difference in the predicted direction in Experiment 1, but not in Experiment 2. Similarly, the average number of test trials obtained in quiet was slightly greater than in the distraction-masker conditions in Experiment 1, but not in Experiment $2 .^{4}$ Thus, there is little evidence for a general effect of the masker on infant behavior.

\section{GENERAL DISCUSSION}

\section{Factors Influencing Infant \\ Masked Thresholds}

The major finding of the current study is that 6-monthold infants require a higher signal level to detect a tone when a second sound is simultaneously presented, even when the second sound does not mask the tone in the traditional sense of peripheral interference. Adults exhibit no threshold elevation under the same conditions, and given the data showing that 6-month-olds have more or less mature auditory filter widths (Olsho, 1985; Schneider et al., 1990 ), it is unlikely that any peripheral effect could have occurred. If the masking effect were due to wider auditory filters, the infants' filters would have to be more than $3000 \mathrm{~Hz}$ wide, an order of magnitude wider than adults'. Moreover, although Schneider et al. (1989) demonstrated that infant masked thresholds increase by $10 \mathrm{~dB}$ when the 
Table 1

Mean and Standard Deviation of General Characteristics of Infant Test Sessions in Quiet and in Distraction Conditions in Two Experiments

\begin{tabular}{|c|c|c|c|c|c|c|}
\hline \multirow[b]{2}{*}{ Condition } & \multicolumn{2}{|c|}{$\begin{array}{l}\text { Standard Deviation } \\
\text { of Reversals }\end{array}$} & \multicolumn{2}{|c|}{$\begin{array}{c}\text { Trials to Training } \\
\text { Criteria }\end{array}$} & \multicolumn{2}{|c|}{ Test Trials } \\
\hline & $M$ & $S D$ & $M$ & $S D$ & $M$ & $S D$ \\
\hline \multicolumn{7}{|c|}{ Experiment 1} \\
\hline Quiet & 1.8 & 1.4 & 14.7 & 4.0 & 41.8 & 9.8 \\
\hline 40-dB noise & 1.6 & 1.0 & 18.8 & 6.0 & 36.1 & 4.7 \\
\hline 50- $\mathrm{dB}$ noise & 1.9 & 1.0 & 18.8 & 4.8 & 37.0 & 8.9 \\
\hline \multicolumn{7}{|c|}{ Experiment 2} \\
\hline Quiet & 1.9 & 1.1 & 28.7 & 11.3 & 29.5 & 14.1 \\
\hline 40- $\mathrm{dB}$ noise & 1.9 & 0.9 & 27.9 & 13.8 & 30.3 & 12.2 \\
\hline
\end{tabular}

masker spectrum level was increased by $10 \mathrm{~dB}$, as is the case for adults (Hawkins \& Stevens, 1950), distraction masking does not appear to increase with masker level in the same way. Finally, infants do not appear to habituate to or learn to ignore the distraction masker when it is presented continuously during a test session.

Schneider et al. (1989) considered four explanations for the elevation of masked thresholds commonly observed in infants and children. They concluded that changes in the neural representation of intensity must be responsible, dismissing the mechanical efficiency of the ear, auditory filter width, motivation and attention, and variability in the neural representation as possible factors. We agree that mechanical efficiency and changes in auditory filter width cannot be responsible for age-related changes in masked threshold. We also agree that at this time no data speak to the issue of variability in neural representation. Whether or not nonlinear changes in the neural representation do occur remains to be shown. However, the results of the current study demonstrate that changes in attention cannot be dismissed as a contributor to elevated infant masked thresholds.

It is important to note that our definition of attentional effects differs considerably from the implicit definition offered by Schneider et al. (1989), or for that matter by Olsho, Koch, and Halpin (1987). Earlier papers have considered "inattentiveness" to mean that the listener has essentially no information about the stimulus on a certain proportion of trials and is forced to guess on those trials. "Inattentiveness" is considered something that can be observed by watching a listener: "we have observed little inattentiveness at any age, except for 6.5-month-olds," and "4- and 8-year-olds appeared to be highly motivated, trying hard to detect the location of the stimulus, and occasionally becoming upset when they made errors" (Schneider et al., 1989, p. 1738). In other words, the listener who appears to be listening to the stimulus is held to be attentive, but the listener who appears to listen to or look at something else is held to be inattentive. Even if one limits the definition of attention to this sort of process, there seems to be a consensus that inattentiveness, as indicated by casual observation or shallow psy- chometric function slopes (Olsho, Koch, \& Halpin, 1987; Schneider et al., 1989), does occur in infants.

On the other hand, here we define attention as a cognitive process whereby the perceiver selects some inputs for further processing or allocates processing resources to some task. A wealth of evidence supports the contention that attention in this sense develops substantially between infancy and adulthood (Flavell, 1985; Olson \& Sherman, 1983); little or no evidence suggests that the ability to attend selectively in this way is something a child can control by trying hard. Thus, much of the controversy over the importance of attentional factors in psychophysical performance of infants and children appears to result from confusion over what is meant by "attention."

By the present definition, then, the results of this study suggest that 6-month-old infants have difficulty in selecting one sound for processing when another sound is presented simultaneously or difficulty in allocating processing resources to optimize detection of one sound. We have no evidence that bears on whether this difficulty is responsible for elevation of masked thresholds in older children, and it is possible that different processes account for changes in performance in different age ranges. This notion is certainly consistent with the general flavor of much developmental theory (e.g., Flavell, 1985; Piaget, 1954).

\section{Factors Influencing the \\ Amount of Masking in Infants}

The discussion thus far has been couched in terms of the factors contributing to age-related changes in masked threshold. However, it can be argued on several grounds that the amount of masking, the difference between the masked and unmasked thresholds for the same signal, is a more appropriate metric. First, if the amount of masking remains constant over age, then a parsimonious explanation for the threshold elevation is one that can account for the elevation of both masked and unmasked thresholds. This is true whether the underlying factors are properties of the sensory system, of nonsensory processes, or both. Second, procedural differences across laboratories would be expected to influence both masked and un- 
masked thresholds; thus, amount of masking is a sounder basis of comparison across studies. Finally, given that there is considerable variability in performance among infants, amount of masking may provide a measure that controls for variation in sensitivity or in other processes.

There is essentially no disagreement about infant masked thresholds' being higher than those of adults. Surprisingly, if one asks whether the amount of masking seen in infants differs from that seen in adults, one will find that the results are much less consistent across studies. Schneider et al. (1989), for example, report 10-20 dB less masking in 6.5-month-olds than in adults for frequencies between 0.4 and $4 \mathrm{kHz}$, but no age difference in amount of masking at $10 \mathrm{kHz}$. Nozza and Wilson (1984) report about $5 \mathrm{~dB}$ less masking in 6-month-olds at $1 \mathrm{kHz}$ and no difference in the amount of masking at $4 \mathrm{kHz}$. Olsho (1985), finally, reports 11-16 dB more masking in 6month-olds for frequencies between 0.5 and $4 \mathrm{kHz}$.

There are many differences among the stimuli and procedures used in these studies, but there is no particular reason to expect that the differences between psychophysical methods would differentially affect masked and unmasked thresholds. However, examination of the unmasked and masked thresholds in these three studies shows that the difference between infants and adults in masked threshold between studies ranges over $10 \mathrm{~dB}$, while the infant-adult difference in unmasked threshold ranges over $18 \mathrm{~dB}$. Moreover, the study showing the least amount of masking in infants has the highest unmasked thresholds relative to those of adults (Schneider et al., 1989), and the study showing the greatest amount of masking in infants has the lowest unmasked thresholds relative to those of adults (Olsho, 1985). Thus, the betweenstudy differences in amount of masking are due more to differences in unmasked threshold than they are to differences in masked threshold.

There are several plausible explanations for the between-study variability in unmasked thresholds. First, Nozza and Wilson (1984) screened their subjects by tympanometry on the day of the test to eliminate infants with middle-ear dysfunction. Trehub, Schneider, and Endman (1980), in the study from which the unmasked thresholds in Schneider et al. (1989) were taken, did not. Although Olsho (1985) did not use tympanometry to screen subjects, subsequent analyses in the same laboratory have shown that although infants who fail tympanometry following the test session are likely to complete the training procedure during their test session, they do not produce acceptable threshold data by our criteria (Ward \& Werner, 1991). Since Trehub et al. (1980) did not exclude subjects who completed the test series of 20 trials, no matter what their performance on these trials, it is likely that their sample included some subjects with middle-ear effusion. Since middle-ear dysfunction would affect the transmission of both signal and masker, it would not be expected to affect masked thresholds. In fact, Schneider et al. (1989) argue that mechanical efficiency of the ear would be expected to affect unmasked thresholds but not masked thresholds, and they offer this as a likely explanation of the increase in masking with age that they have observed. Some would question whether middle-ear effusion is a developmental effect in the usual sense of the term, but it does affect the mechanical efficiency of the ear.

Second, both Nozza and Wilson (1984) and Olsho (1985) presented their stimuli over earphones, whereas Trehub et al. (1980) used sound-field presentation. Olsho, Koch, Carter, Halpin, and Spetner (1988), in a comparison of studies of infant absolute sensitivity, showed that studies with earphones produced lower infant thresholds, relative to those of adults, than did studies with sound-field presentation. If such effects are due to the additional attenuation of ambient noise provided by the earphone cushion, masked thresholds would not be similarly affected.

Finally, the fact that Nozza and Wilson (1984) and Olsho (1985) made unmasked-masked comparisons in the same subjects, but that Schneider et al. (1989) compared thresholds across different studies, must be considered. In studies that have estimated individual thresholds, infant thresholds are always more variable than those of adults (e.g., Berg \& Smith, 1983; Nozza \& Wilson, 1984; Olsho, Koch, et al., 1987; Sinnott, Pisoni, \& Aslin, 1983). Thus, the potential for differences between samples is greater; it is thus possible that Trehub et al. (1980) simply had a less sensitive sample of infants or that for nonobvious reasons, infant performance was poorer in that study.

At this point, then, it seems premature to come to any conclusion about the development of the amount of masking. Additional work to address the development of auditory attention and to control for factors such as middleear status and between-subjects variability is clearly needed.

\section{REFERENCES}

Allen, P., Wightman, F., Kistler, D., a Dolan, T. (1989). Frequency resolution in children. Joumal of Speech \& Hearing Research, 32, 317-322.

BerG, K. M., SMITH, M. C. (1983). Behavioral thresholds for tones during infancy. Journal of Experimental Child Psychology, 35, $409-425$.

Flavell, J. H. (1985). Cognitive development (2nd ed.). Englewood Cliffs, NJ: Prentice-Hall.

Fletcher, H. (1940). Auditory patterns. Reviews of Modern Physics, $12,47-65$.

Green, D. M. (1988). Profile analysis: Auditory intensity discrimination. New York: Oxford University Press.

Green, D. M., Swets, J. A. (1966). Signal detection theory and psychophysics. New York: Wiley.

HawkINS, J. E. , STeveNs, S. S. (1950). The masking of pure tones and of speech by white noise. Joumal of the Acoustical Society of America, 22, 6-13.

Jensen, J. K., NefF, D. L., Callaghan, B. P. (1989, October). Discrimination of intensity, frequency, and duration discrimination in young children. Paper presented to the American Speech-HearingLanguage Association, New Orleans.

NeFF, D. L., Green, D. M. (1987). Masking produced by spectral uncertainty with multicomponent maskers. Perception \& Psychophysics, 41, 409-415.

NozzA, R. J., WiLson, W. R. (1984). Masked and unmasked pure- 
tone thresholds of infants and adults: Development of auditory frequency selectivity and sensitivity. Journal of Speech \& Hearing Research, 27, 613-622.

OLsho, L. W. (1985). Infant auditory perception: Tonal masking. Infant Behavior \& Development, 8, 371-384.

Olsho, L. W., Кoch, E. G., Carter, E. A., Halpin, C. F., SPeTNER, N. B. (1988). Pure-tone sensitivity of human infants. Joumal of the Acoustical Society of America, 84, 1316-1324.

Olsho, L. W., Косн, E. G., HalPIN, C. F. (1987). Level and age effects in infant frequency discrimination. Journal of the Acoustical Society of America, 82, 454-464.

Olsho, L. W., Косh, E. G., HAlPiN, C. F., CARTer, E. A. (1987). An observer-based psychoacoustic procedure for use with young infants. Developmental Psychology, 23, 627-640.

Orson, G. M., Sherman, T. (1983). Attention, learning, and memory in infants. In M. M. Haith \& J. J. Campos (Eds.), Handbook of child psychology: Vol. 2. Infancy and developmental psychobiology (pp. 1001-1080). New York: Wiley.

Patterson, R. D., Nimmo-Smith, l., Weber, D. L., \& Milroy, R. (1982). The deterioration of hearing with age: Frequency selectivity, the critical ratio, the audiogram, and speech threshold. Journal of the Acoustical Society of America, 72, 1788-1803.

Piaget, J. (1954). The construction of reality in the child. New York: Basic Books.

SANES, D. H., Rubel, E. W. (1988). The development of stimulus coding in the auditory system. In A. F. Jahn \& J. Santos-Sacchi (Eds.), Physiology of the ear (pp. 431-455). New York: Raven Press.

Schneider, B. A., Morrongiello, B. A., Trehub, S. E. (1990). Size of critical band in infants, children, and adults. Journal of Experimental Psychology: Human Perception \& Performance, 16, 642-652.

SChneider, B. A., Trehub, S. E., Morrongiello, B. A., \& Thorpe, L. A. (1989). Developmental changes in masked thresholds. Journal of the Acoustical Society of America, 86, 1733-1742.

SinNotT, J. M., \& AsLIN, R. N. (1985). Frequency and intensity discrimination in human infants and adults. Journal of the Acoustical Society of America, 78, 1986-1992.

Sinnotr, J. M., Pisoni, D. B., Asun, R. M. (1983). A comparison of pure tone auditory thresholds in human infants and adults. Infant Behavior \& Development, 6, 3-17.

SPETner, N. B., \& OLSho, L. W. (1990). Auditory frequency resolution in human infancy. Child Development, 61, 632-652.

Taylor, M. M., CReelman, C. D. (1967). PEST: Efficient estimates on probability functions. Journal of the Acoustical Society of America, 41, 782-787.

Trehub, S. E., Schneider, B. A., Endman, M. (1980). Developmental changes in infants' sensitivity to octave-band noises. Journal of Experimental Child Psychology, 29, 282-293.

Veloso, K., Hall, J. W., III, \& Grose, J. H. (1990). Frequency selectivity and comodulation masking release in adults and 6-year-old children. Journal of Speech \& Hearing Research, 33, 96-102.

W ARD, J. H., \& WERNER, L. A. (1991, February). The effect of otitis media on the threshold of hearing in infants. Paper presented to the Midwinter Research Meeting of the Association for Research in Otolaryngology, St. Petersburg Beach, FL.

Watson, C. S., Kelly, W. J., \& Wroton, H. W. (1976). Factors in the discrimination of auditory patterns. II. Selective attention and learning under various levels of stimulus uncertainty. Joumal of the Acoustical Society of America, 60, 1176-1186.

WEGEL, R. L., LANE, C. E. (1924). The auditory masking of one sound by another and its probable relation to the dynamics of the inner ear. Physical Review, 23, 266-285.

Werner, L. A., Marean, G. C. (in press). Methods for estimating infant thresholds. Journal of the Acoustical Society of America.

\section{NOTES}

1. The thresholds obtained from infants in the present study are higher than those that we have reported in the past (e.g., Olsho, Koch, Carter, Halpin, \& Spetner, 1988). We believe that several factors contribute to this difference. First, the psychophysical procedure used here is different, and all other things being equal, it would be expected to produce higher thresholds. Second, our criteria for accepting thresholds were stricter here. Third, there is variability among observers in the observerbased psychoacoustic procedure with respect to the thresholds they obtain from infants; two of the three observers who tested the infants in the present study generally tend to get thresholds in the higher end of the range. However, there is no evidence that these factors interact with distraction-masker condition. Thus, we do not believe that they contribute to the quiet-masked differences reported here.

2. Since the hypothesis in all these comparisons was that the distractionmasking threshold would be higher than the quiet threshold, we conducted one-tailed tests. However, if we had used a two-tailed test, the adult threshold in the presence of the distraction masker would have been significantly better than the threshold in quiet $[t(10)=3.59, p=$ $.005]$. This may suggest that the adults are using some form of profile analysis to perform the task (Green, 1988). Since the purpose of testing adults here is primarily to ensure that peripheral masking effects are minimal, the fact that this difference arose does not affect the interpretation of the infant data.

3. We also calculated $\beta$, a quantitative measure of bias from the data shown in Figure 4. In Experiment 1, mean $\log \beta$ in the quiet condition was $-0.05(S D=0.16)$, and in the $40-\mathrm{dB}$ distraction-masker condition, it was $-0.10(S D=0.14)$. A paired-sample $t$ test on mean log $\beta$ was not significant $[t(8)=0.91, p>.10]$. In Experiment 2 , mean $\log \beta$ was $0.03(S D=0.16)$ in the quiet condition and $0.03(S D=0.19)$ in the 40 - $\mathrm{dB}$ distraction-masker condition. Although these quantitative results lend credence to the conclusion that a criterion shift is not responsible for the distraction-masking effect, they should be interpreted with caution: $\beta$ is a parametric statistic, and it is not known whether the parametric assumptions underlying its use are justified in a procedure such as that used here.

4. As noted above, it was more difficult to obtain thresholds for infants in the distraction-masker conditions, particularly when the distraction masker level was $50 \mathrm{~dB}$ SPL. In the analyses described here, only infants in the final sample are included. While it is possible that the distraction masker had a less specific effect on infants in general, the infants included in the current analysis showed the distraction-masking effect but did not show general effects of the masker.

(Manuscript received March 28, 1991; revision accepted for publication July 15,1991 .) 